\title{
Biodegradação de polietileno de alta densidade por meio de larvas e insetos de Galleria mellonella (Linnaeus, 1758) (Lepidoptera: Pyralidae)
}

\section{Lucas Vinícius Rodrigues Godoi*, Evandro de Castro, Felipe Minoru Inagaki, José Hilton Bernardino de Araújo}

Universidade Tecnológica Federal do Paraná. Campo Mourão. Rodovia BR-369, km 0,5. Saída para Cascavel. Campo Mourão-PR, Brasil (CEP 87301-006). *E-mail: luukaaz_@live.com.

Resumo. Com o consequente crescimento populacional, tem-se o alto consumo de recursos naturais ocasionando elevação da geração de resíduos sólidos e sua incorreta disposição, que gera grandes problemas ambientais. Um dos geradores desses problemas é o plástico polietileno. Tendo em vista a quantidade de resíduo plástico gerado, e a dificuldade deste de se biodegradar, tornam-se necessárias novas soluções para a degradação do plástico. Assim, a utilização de Galleria mellonella (Linnaeus, 1758) (Lepidoptera: Pyralidae) torna-se uma alternativa. Diante disto, realizou-se um experimento a fim de verificar a hipótese do polietileno ser biodegradado por meio de indivíduos nos estágios larval e adulto (mariposas) do inseto G. mellonella. Utilizou-se mariposas em estágio adulto e em estágio larval diretamente sobre amostras de sacolas plásticas feitas de Polietileno de Alta Densidade (PEAD), e também com mel para incentivar o consumo do polietileno durante um período de 28 dias. Após o período de estudo podemos constatar o consumo do polietileno pelos insetos de G. mellonella. Ao depararse com a biodegradação do PEAD pela espécie estudada, foi obtido um resultado positivo, no entanto o experimento apresentou números baixos de biodegradação, sendo de $0,5 \%$ para o caso de uso de mariposas adultas no período de 28 dias. Diante deste fato evidencia-se a necessidade de realizar maiores estudos e pesquisas relacionadas.

Palavras-chave: Degradação; Larvas; Mariposa; PEAD; Plástico.

Abstract. Biodegradation of high density polyethylene by larvae and insects of Galleria mellonella (Linnaeus, 1758) (Lepidoptera: Pyralidae). With the consequent population growth, one has the high consumption of natural resources causing the generation of large amounts of work and its integrity. One of the drivers of the problems is plastic polyethylene. In view of a part of the vibration, the new generation, and its difficulty to biodegrade, new solutions for a degradation of the plastic become necessary. Thus, the use of Galleria mellonella (Linnaeus, 1758) (Lepidoptera: Pyralidae) becomes an alternative. Thus, a process

Recebido:

16/07/2018

Aceito:

25/08/2018

Publicado:

31/08/2018

Acesso aberto

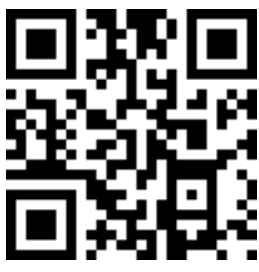

ORCID

(1) 0000-0001-8907-6631

Lucas Vinícius

Rodrigues Godoi

(D) 0000-0003-4698-8002 Evandro de Castro

(D) 0000-0001-7300-2857 Felipe Minoru Inagaki (D) 0000-0001-9743-7855 José Hilton Bernardino de Araújo 
of verification of a polyethylene hypothesis was produced and biodegraded by means of an individual in the larval and adult stages (moth) of the insect G. mellonella. It was used orange and adult moths directly on samples of plastic bags made of High Density Polyethylene (HDPE), and also for the purpose of consuming polyethylene for a period of 28 days. After the period of study, we can verify the consumption of polyethylene by the insects of $G$. mellonella. To be faced with the biodegradation of HDPE by the series of studies, being at the moment positive, however the experiment in every day was of biodegradation, being of $0.5 \%$ for the case of use of adult moths in the period of 28 days. Faced with this fact, the need to carry out more studies and related research is evident.

Keywords: Degradation; Larvae; Greater wax moth; HDPE; Plastic.

\section{Introdução}

No Brasil são produzidos cerca de três milhões de toneladas de plásticos anualmente, e o polietileno que é matéria-prima para a produção de sacolas plásticas leva em média 500 anos para se decompor quando descartado como resíduo (Oliveira et al., 2012; Portal Resíduos Sólidos, 2013).

O polietileno é um polímero parcialmente cristalino, flexível, cujas propriedades são acentuadamente influenciadas pela quantidade relativa das fases amorfa e cristalina. O Polietileno de Alta Densidade (PEAD) é utilizado em diferentes segmentos da indústria de transformação de plásticos (Coutinho, 2003). Sendo o de maior produção mundial, alcançando em 2017 o volume aproximado de 30 milhões de toneladas e no Brasil, a produção em 2008 alcançou aproximadamente 925.000 toneladas (Mesquita, 2010).

Diante de tais fatos citados acima, há a necessidade de encontrar uma solução ambiental e economicamente viável de degradar o polietileno. Recentemente foi publicado uma pesquisa elaborada por Bombelli et al. (2017) demonstrando resultados da biodegradação do polietileno obtida ao utilizar o inseto Galleria mellonella (Linnaeus, 1758) (Lepidoptera: Pyralidae), e através da relevância científica de tal estudo, decidimos realizar este experimento utilizando método diferente.

Esta pesquisa tem como objetivo analisar, acompanhar e medir a biodegradação de sacolas plásticas compostas por PEAD, por meio de indivíduos nos estágio larval e adulto (mariposa) do inseto G. mellonella.

\section{Metodologia}

0 experimento foi realizado no Laboratório de Processos Industriais, da Universidade Tecnológica Federal do Paraná, no Campus Campo Mourão. Foram utilizados recipientes plásticos em quatro situações diferentes, vedados com plástico filme, com aberturas mínimas para que os indivíduos pudessem respirar. 0 primeiro recipiente (P1) com duas mariposas em estágio adulto com um recorte $(5 \mathrm{~cm} \times 5 \mathrm{~cm})$ de uma sacola, no segundo recipiente (P2) foram colocados dois insetos dentro de uma sacola fechada, no terceiro recipiente (P3) foi colocada uma larva em uma sacola fechada, quanto ao quarto recipiente (P4) foi colocada uma larva junto a um recorte de sacola $(5 \mathrm{~cm} \times 5 \mathrm{~cm})$, e após sete dias foram acrescentadas mais duas larvas.

Todas as sacolas e recortes foram pesados antes e depois dos experimentos, com o objetivo de 
quantificar a biodegradação dos materiais. 0 experimento foi monitorado em um intervalo de sete dias durante 28 dias, com o intuito de observar e coletar dados.

\section{Resultados e discussão}

Os indivíduos de G. mellonella, conhecidos popularmente como traça do mel, foram pesados ao longo de quatro semanas em recipientes com sacolas plásticas inteiras e recortes de $5 \mathrm{~cm} \times 5 \mathrm{~cm}$, tanto indivíduos adultos como em fase larval, com um intervalo de sete dias para cada pesagem. Os resultados encontrados ao longo das semanas de pesagem podem ser observados na Tabela 1.

Tabela 1. Relação do peso dos experimentos durante as quatro semanas de análise.

\begin{tabular}{lcccc}
\hline Experimento & 1 $^{\text {a Semana }}$ & 2 $^{\text {a Semana }}$ & 3 $^{\text {a Semana }}$ & 4 $^{\text {a Semana }}$ \\
\hline P1 & 0,0477 & 0,0477 & 0,0477 & 0,0477 \\
P2 & 0,0504 & 0,0504 & 0,0502 & 0,0502 \\
P3 & 4,9072 & 4,8940 & 4,8860 & 4,8855 \\
P4 & 1,2522 & 1,2520 & 1,2520 & 1,2520 \\
\hline
\end{tabular}

No início do experimento, utilizou-se quatro mariposas em estágio adulto e duas em estágio larval, contudo, na segunda semana houve uma mortalidade expressiva das mariposas no estágio adulto, dentre as quatro, apenas duas sobreviveram. Desta forma, utilizou-se mel no experimento 1 e 2, para que incentivasse tanto a mariposa quanto a larva a comerem o polietileno.

Houve consumo do polietileno pelas larvas, visto que a quantidade consumida da primeira a quarta semana foi de 0,0219 g no total, sendo possível ser verificado na Figura 1 o material antes de ser degradado e na Figura 2, após apresentar a degradação induzida.

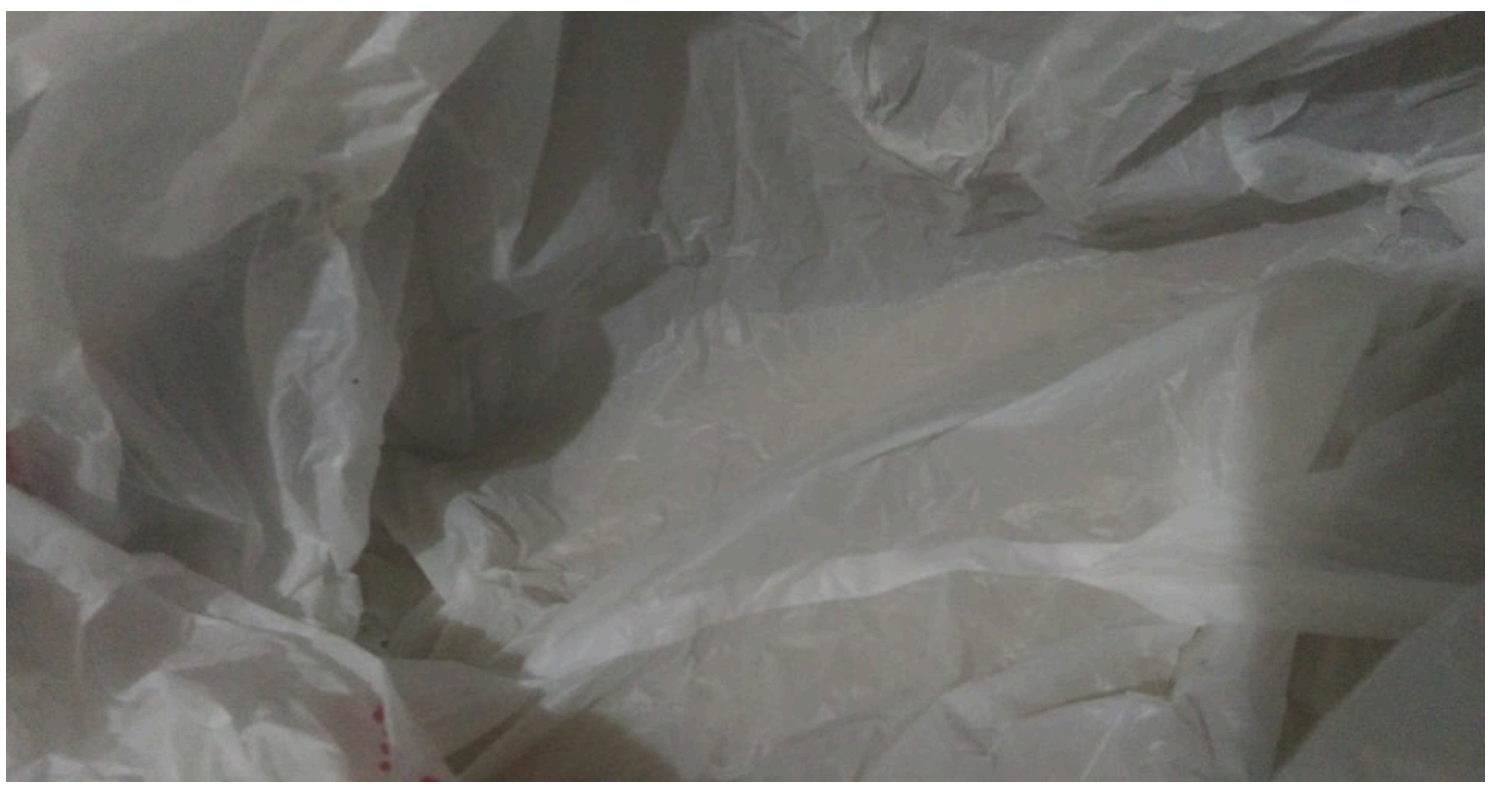

Figura 1. Exemplar de polietileno de alta densidade utilizado no experimento. 
Destaca-se que na primeira semana o peso total dos plásticos era
$6,2575 \mathrm{~g}$, enquanto que na quarta semana o peso total foi de $6,2354 \mathrm{~g}$.

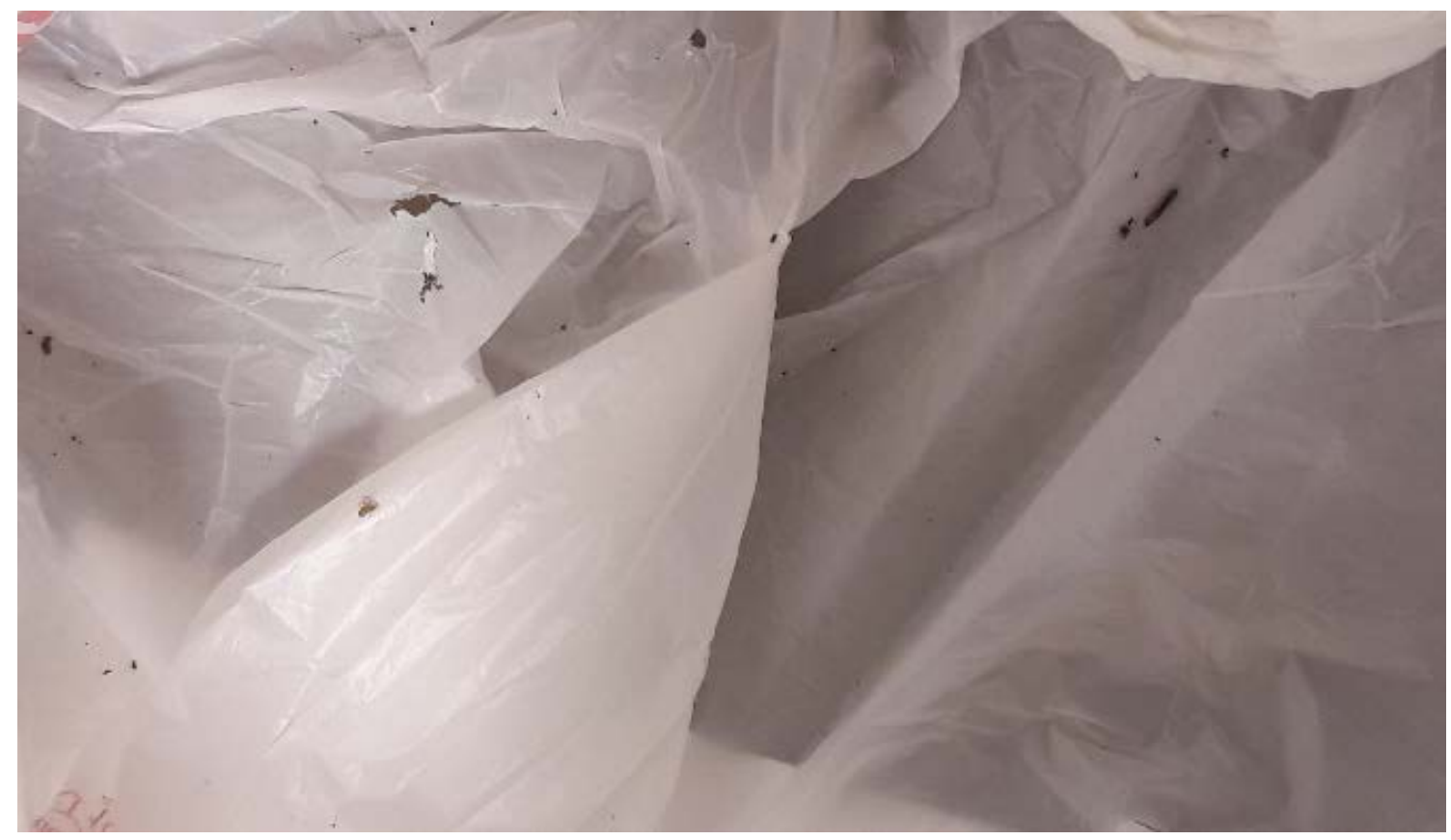

Figura 2. Polietileno de alta densidade biodegradado por larvas de G. mellonella.

Foi verificado que os indivíduos de Galleria mellonella em fase larval consumiram mais o plástico do que os indivíduos adultos, degradando $12,5 \%$ a mais do que os indivíduos em fase adulta, como constatado na Figura 3.

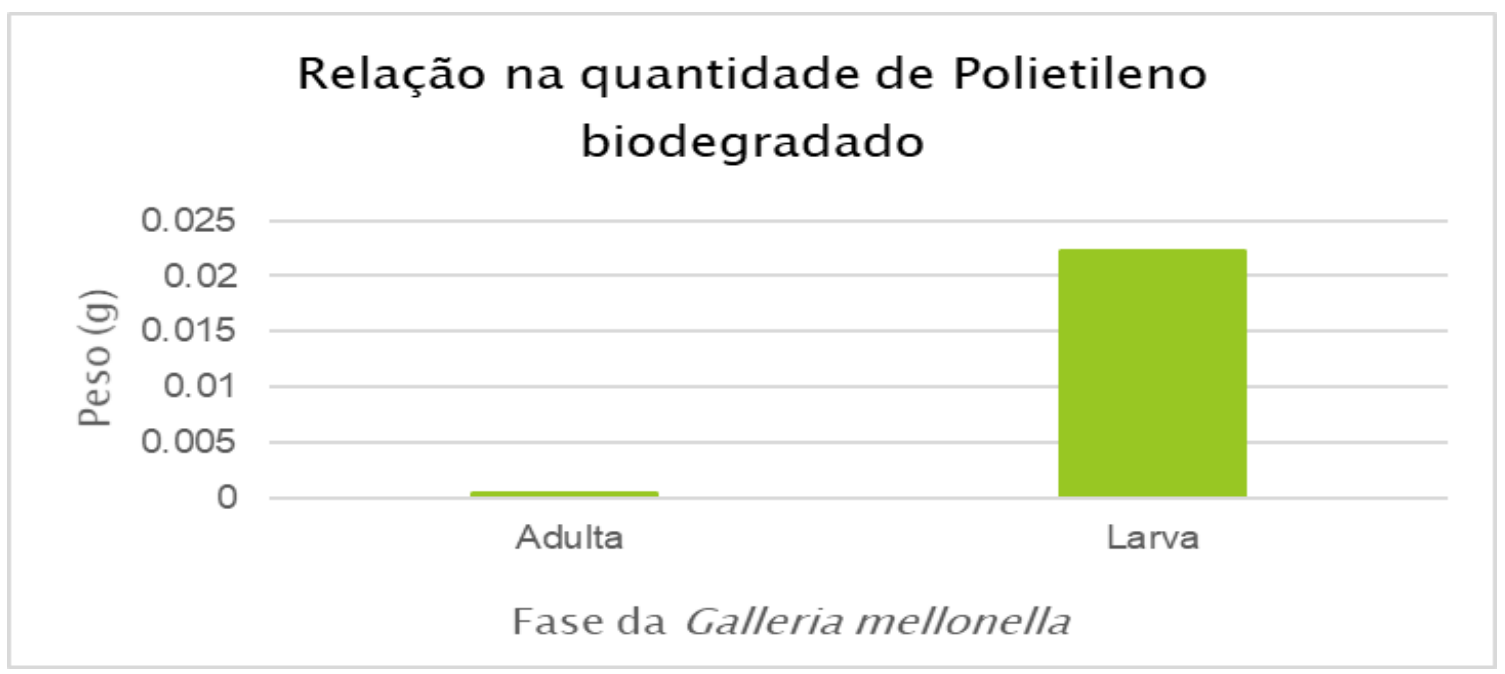

Figura 3. Relação na quantidade de polietileno biodegradado. 
0 crescimento da larva foi acompanhado por 28 dias, entretanto, não foi constatado desenvolvimento de sua estrutura, levando a conclusão de que os indivíduos não tiveram condições ideais para chegarem à sua fase adulta $\mathrm{e}$, assim, proliferar conforme está apresentado na Figura 4.

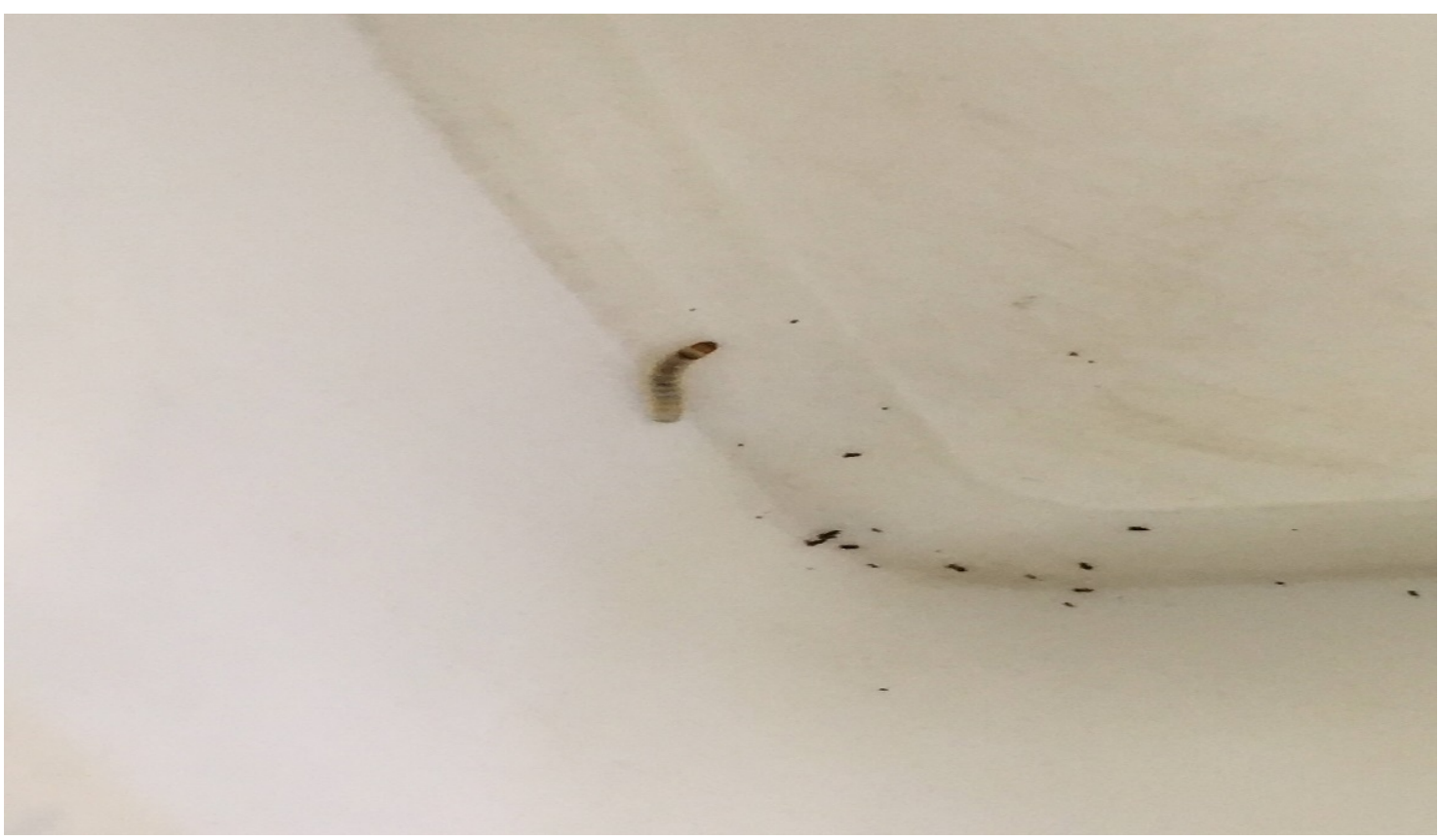

Figura 4. Indivíduo em estágio larval de G. mellonella.

Tal resultado pode ser devido a diversos fatores que combinados ou isolados foram determinantes para a falta de desenvolvimento do ser vivo, como por exemplo a falta de nutrientes que a espécie necessitava, bem como a alta complexidade de biodegradação das sacolas plásticas constituídas por PEAD (polietileno de alta densidade).

Segundo Ramarao et al (2012), são necessárias duas semanas para a evolução do último estágio larval à mariposa adulta. Os machos adultos medem de 10 a $15 \mathrm{~mm}$ e possuem cor bege e marcas escuras, enquanto as fêmeas adultas medem em torno de 20 mm e são mais escurar que os machos, possuindo cor marrom ou cinza.

Pode-se observar que os indivíduos encontrados em fase adulta, segundo a definição de Ramarao (2012) trata-se de fêmeas (Figura 5). Tal constatação pode justificar a não proliferação da espécie pela falta de um indivíduo macho para acasalar e reproduzir. 


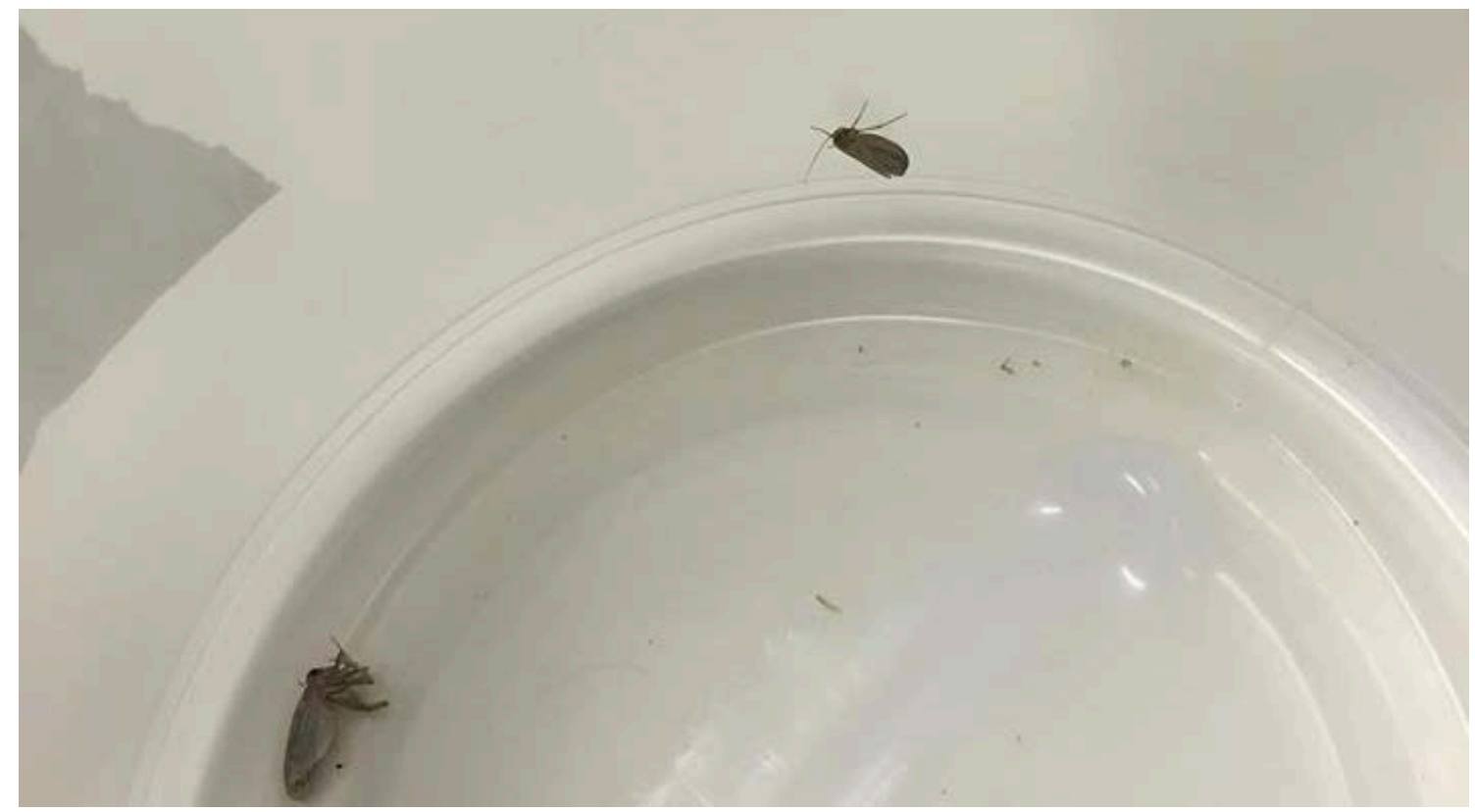

Figura 5. Indivíduos em estágio adulto de G. mellonella.

\section{Conclusão}

O polietileno é um polímero altamente utilizado devido suas características e propriedades. Tal fato faz com que este seja largamente utilizado e, desta forma, acumulado no planeta. Com o trabalho desenvolvido, pode-se observar que há uma carência de trabalhos brasileiros realizados com a espécie G. mellonella na biodegradação do PEAD.

A espécie utilizada não apresentou desenvolvimento ao alimentar-se com o substrato em questão. Porém, não é possível afirmar a causa do ocorrido. A falta de nutrientes pode ser fator que determinou 0 resultado presente neste trabalho.

Ao deparar-se com a biodegradação do PEAD da espécie estudada, foi obtido um resultado positivo, no entanto o experimento apresentou números baixos de biodegradação, diante deste fato evidencia-se a necessidade de realizar maiores estudos e pesquisas relacionadas.

\section{Conflito de interesses}

Os autores declaram não haver conflito de interesses.

\section{Referências}

Bombelli, P; Howe, C, J; Bertocchini, F. Polyethylene bio-degradation by caterpillars of the wax moth Galleria mellonella. Current Biology, v. 27, n. 8, p. 283-293, 2017. https://doi.org/10.1016/j.cub.2017.02.060

Branco, A. Larva da farinha come plástico e excreta resíduo biodegradável. 2015. Disponível em: <https://www.greenme. com.br/informar-se/lixo-e-reciclagem/2336larva-da-farinha-come-plastico-e-excretaresiduo-biodegradavel $>$. Acesso em: 20 nov. 2017.

Coutinho, F. M. B.; Mello, I. L.; Santa Maria, L. C. Polietileno: principais tipos, propriedade e aplicações. Polímeros: Ciência e Tecnologia, v. 13, n. 1, p. 1-13, 2003. https://doi.org/10.1590/S0104-142820030 00100005

Ferro, E.S. Biotecnologia translacional: hemopressina e outros peptídeos intracelulares. Estudos Avançados, v. 24, n. 70, p. 109-121, 2010. https://doi.org/ 10.1590/s0103-40142010000300008 
Machado, G. Reciclagem de plásticos. 2013. Disponível em: <https://portalresiduos solidos.com/reciclagem-de-plasticospolimeros/>. Acesso em: 02 maio 2018.

Mesquita, F. A. Modificação das propriedades do polietileno de alta densidade por diferentes condições de extrusão. São Paulo, Universidade de São Paulo, 2010. (Dissertação de mestrado). https://doi.org/10.11606/D.3.2010.tde10012011-103025

Monteiro, M. A.; Silva, E. S.; Souza, J. G. Plano de gerenciamento integrado de resíduos de isopor - PGIRI. Belo Horizonte: Fundação Estadual do Meio Ambiente, 2011.

Oliveira, L.; Lacerda, C. S.; Alves, I. J. B. R.; Santos, E. D.; Oliveira, S. A.; Batista, T. S. A. Impactos ambientais causados pelas sacolas plásticas: o caso Campina Grande, PB. Revista Brasileira de Biologia e Farmácia, v. 7, n. 1, p. 88-104, 2012. Disponível em: <http://sites.uepb.edu.br/biofar/download/ v7n1-2012/impactos_ambientais_causados_ pelas_sacolas_plasticas.pdf>. Acesso em: 02 maio 2018.
Ramarao, N.; Nielsen-Leroux, C.; Lereclus, D. The insect Galleria mellonella as a powerful infection model to investigate bacterial pathogenesis. Journal of Visualized Experiments: JoVE, n. 70, e4392, 2012. https://doi.org/10.3791/4392

Vinhas, G. M.; Almeida, Y. M. B.; Lima, M. A. G. A. Estudo das propriedades e biodegradabilidade de blendas de poliéster/ amido submetidas ao ataque microbiano. Química Nova, v. 30, n. 7, p. 1584, 2007. Disponível em: <http://quimicanova.sbq. org.br/imagebank/pdf/Vol30No7_1584_15AR06317.pdf>. Acesso em: 02 maio 2018. 